\title{
MAGNETIC PROPERTIES AND GMR OF SPUTTERED PERMALLOY/Au MULTILAYERS
}

\author{
B. SZYMAŃSKI AND F. STOBIECKI \\ Institute of Molecular Physics, Polish Academy of Sciences \\ Smoluchowskiego 17, 60-179 Poznań, Poland
}

\begin{abstract}
We report on structural, magnetic, and GMR properties of permalloy/Au multilayers where permalloy $=\mathrm{Ni}_{83} \mathrm{Fe}_{17}$, deposited by face-to-face sputtering onto $\mathrm{Si}(111)$ substrate. X-ray diffraction studies confirmed a good structural quality of our multilayers. The samples were characterised with vibrating sample magnetometer, longitudinal magnetooptical Kerr effect and giant magnetoresistance measurements. It was determined that our multilayers are magnetically very soft with $H_{\mathrm{c}} \approx 1 \mathrm{Oe}$ and show uniaxial anisotropy with $H_{K} \approx 5$ Oe. For gold sublayer thickness $d_{\mathrm{Au}}$ close to $1.1 \mathrm{~nm}$ the antiferromagnetic coupling is present in very narrow $A u$ thickness range $(\approx 0.2 \mathrm{~nm})$. Despite a good structural quality of samples relatively small giant magnetoresistance value ( $1.2 \%$ at room temperature) was found. It is due to non-perfect aniferromagnetic coupling caused by pinholes.
\end{abstract}

PACS numbers: 75.70.-i, 75.70.Pa, 68.55.-a

\section{Introduction}

The permalloy $/ \mathrm{Au}(\mathrm{Py} / \mathrm{Au})$ multilayers revealed the greatest field sensitivity of giant magnetoresistance (GMR) [1,2]. It was possible due to a weak exchange coupling ensured by gold and a weak anisotropy field of permalloy. The authors of these papers emphasised that the antiferromagnetic (AF) coupling energy as well as GMR of these multilayers strongly depended on the conditions of deposition. In our earlier works on $\mathrm{Py} / \mathrm{Cu}$ multilayers [3] we have shown that the deposition method of face-to-face sputtering allows much lower values of AF coupling energy than those achieved in multilayers deposited by other techniques. We obtained high sensitivity of GMR, i.e., $S=0.6 \% / 0$ e. The aim of this study was to determine magnetoresistance and magnetic properties of $\mathrm{Py} / \mathrm{Au}$ multilayers with the $\mathrm{Au}$ sublayer thickness from the first region of AF coupling, and to explain the reasons for low values of the GMR amplitude for the multilayers deposited without the buffer layer. 


\section{Experimental}

$\mathrm{A}$ set of $(\mathrm{Py} / \mathrm{Au})_{N}$ multilayers with constant permalloy thickness $d_{\mathrm{Py}}=$ $2.4 \mathrm{~nm}$ and $\mathrm{Au}$ sublayer thickness $d_{\mathrm{Au}}$ ranging from 0.7 to $1.5 \mathrm{~nm}$ and number of repetition $N=50$ was prepared with double face-to-face sputtering [4]. The samples were deposited onto $\operatorname{Si}(111)$ substrate without any buffer layer. The periodic, layered structure of multilayers was confirmed by low and high angle X-ray diffraction (XRD) and modulation wavelength $\Lambda=d_{\mathrm{Py}}+d_{\mathrm{Au}}$ was determined. The XRD studies revealed the polycrystalline structure of the samples with (111) texture. Magnetic hysteresis loops were taken by longitudinal magnetooptical Kerr effect (LMOKE) at room temperature (RT). Independently, magnetisation reversal was measured with vibrating sample magnetometer (VSM) in the temperature range from RT to $200^{\circ} \mathrm{C}$. The a.c. magnetoresistance measurements were performed with the conventional four point method. We define the GMR amplitude as $\Delta R / R=\left(R_{\max }-R_{\min }\right) / R_{\min }$.

\section{Results and discussion}

As follows from the LMOKE measurements carried out at RT for a series of multilayers, the AF coupling occurs for $1.0 \leq d_{\mathrm{Au}} \leq 1.2 \mathrm{~nm}$. Figures 1a and $b$ present exemplary hysteresis loops measured in a magnetic field applied in the plane of the film along or perpendicular to the easy axis of uniaxial anisotropy. From the loops we could determine the anisotropy field $H_{K} \approx 5 \mathrm{Oe}$, the coercive field $H_{\mathrm{c}} \approx 1 \mathrm{Oe}$ and the saturation field $H_{\mathrm{s}} \approx 15$ Oe for the film with the AF coupling shown in Fig. 1a. The coupling constant determined from the value of $H_{\mathrm{s}}$ was $J_{\mathrm{AF}} \approx 3 \mathrm{merg} / \mathrm{cm}^{2}$. It is much lower than that found for the multilayers obtained by the MBE [1] or magnetron sputtering [2] methods. For the sample showing AF coupling, whose hysteresis is shown in Fig. 1a, we measured the value of $\Delta R / R$, which as expected, reaches saturation in a low field.
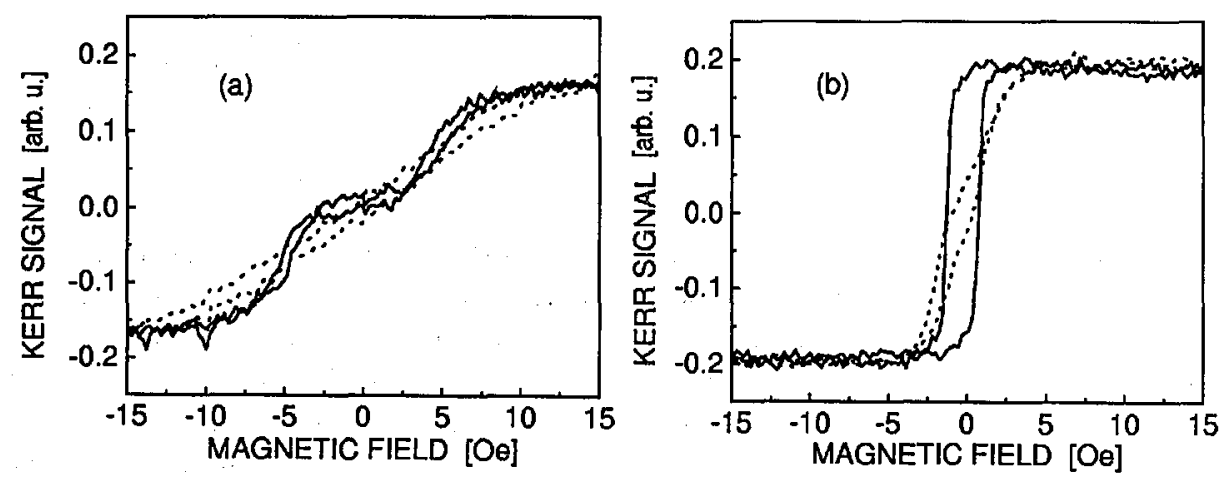

Fig. 1. Magnetooptical hysteresis loops measured at RT for the samples: (a) with antiferromagnetic coupling $-d_{\mathrm{Au}}=1.1 \mathrm{~nm}$ and (b) with ferromagnetic coupling $d_{\mathrm{Au}}=1.3 \mathrm{~nm}$. The solid and dotted lines correspond to magnetic field applied parallel and perpendicular to the easy axis, respectively. 

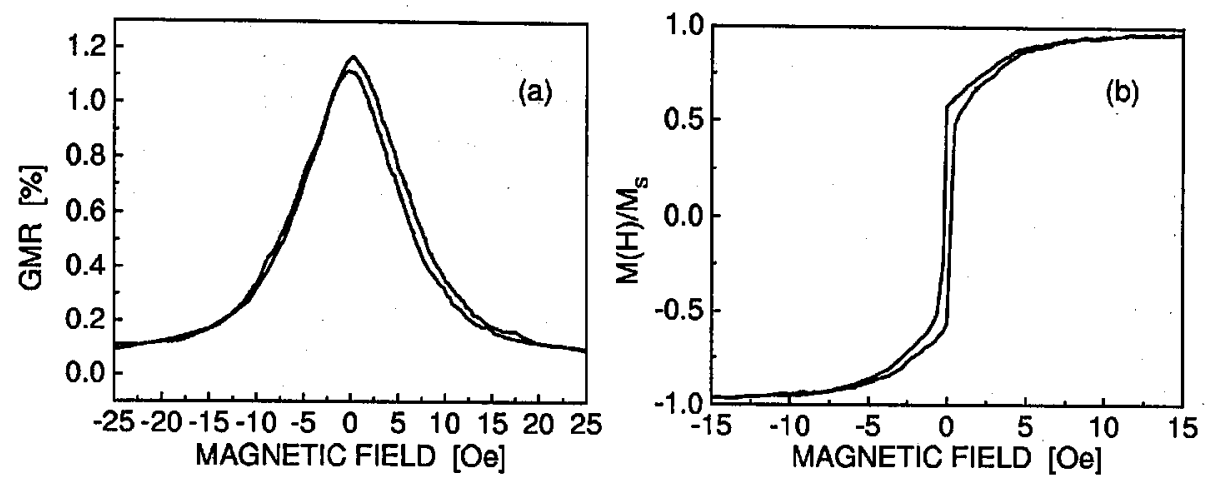

Fig. 2. GMR (a) and VSM magnetisation (b) curves taken at RT for multilayer with $d_{\mathrm{Au}}=1.1 \mathrm{~nm}$ (magnetic field was applied parallel to the easy axis).
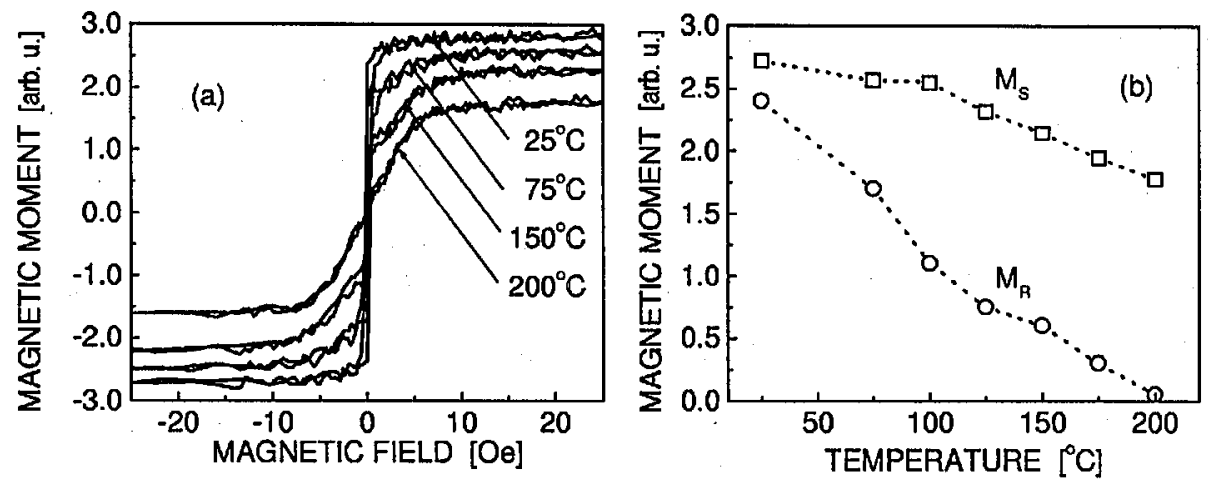

Fig. 3. (a) Temperature changes of magnetisation reversal process for multilayer with $d_{\mathrm{Au}}=1.2 \mathrm{~nm}$ (magnetic field parallel to the easy axis); (b) saturation and remanence magnetisation ( $M_{\mathrm{s}}$ and $M_{\mathrm{r}}$ ) versus temperature for the same sample as in (a).

The relative change in resistance did not exceed the value of $1.2 \%$ (Fig. 2a). Such a small value of the GMR amplitude was the result of a small volume fraction of the film showing the AF coupling, $F_{\mathrm{AF}}=1-M_{\mathrm{r}} / M_{\mathrm{s}}=0.4$, for the layer with $d_{\mathrm{Au}}=1.1 \mathrm{~nm}$ (Fig. 2b). In order to identify the reason for a low value of $F_{\mathrm{AF}}$, the temperature measurements of magnetisation reversal were performed with the use of VSM. To make the temperature changes in the hysteresis loop more pronounced, we have chosen a sample of an even worse $F_{\mathrm{AF}}$ factor ( 0.2 at RT).

As illustrated in Figs. 3a and $\mathrm{b}$, with increasing temperature the value of $M_{\mathrm{r}}$ decreases much quicker than $M_{\mathrm{s}}$, getting close to zero at $T=200^{\circ} \mathrm{C}$, which suggests that almost complete AF coupling has been reached in the whole volume of the multilayer $\left(F_{\mathrm{AF}}=1\right)$. As shown in papers $[5,6]$ such a behaviour is characteristic of multilayer systems with AF coupling, in which the neighbouring magnetic layers locally show a ferromagnetic coupling induced by the pinholes ("magnetic bridges"). The observed effect can be explained by the fact that the 
magnetic efficiency of pinholes, owing to their limited size, with increasing temperature decreases faster than at the rate following from the $M_{\mathrm{s}}(T)$ dependence for permalloy. The differences in the field dependencies of LMOKE and VSM loops (compare Fig. 1a and Fig. 2b) stem from the technique of the signal accumulation in both methods. The LMOKE signal brings the information from the thin surface layer and the region $\approx 2 \mathrm{~mm}^{2}$, whereas the VSM signal contains the information from the whole volume of the sample. As mentioned above, $F_{\mathrm{AF}}$ value estimated from VSM is 0.4 , while the value obtained from LMOKE measurements is 1 , for the same multilayer. It suggests that the density of pinholes, high in the first stage of the sample deposition, decreases with its increasing thickness reaching almost zero in the subsurface region. Therefore, it is expected that the use of an appropriate buffer layer should lead to a significant reduction of the influence of the pinholes on GMR, due to a reduction of their density.

\section{Conclusions}

In this study we have shown that with the use of the face-to-face sputtering technique it is possible to obtain for $d_{\mathrm{Au}} \approx 1.1 \mathrm{~nm}$ low values of $\mathrm{AF}$ coupling (low $H_{\mathrm{s}}$ ), and a linear $R(H)$ dependence without hysteresis. The improvement of the quality of the layers by elimination of pinholes responsible for the local regions showing ferromagnetic coupling may lead to about 2.5 fold increase in the GMR amplitude (taking into account that $\Delta R / R \propto F_{\mathrm{AF}}$ ) and thus to an increase in the field sensitivity $S$ to about $0.3 \% / \mathrm{Oe}$. These modifications will make the $\mathrm{Py} / \mathrm{Au}$ layers attractive from the point of view of the sensor technology.

\section{Acknowledgments}

The measurements were partly performed at the University in Kassel thanks to the financial support by the German-Polish scientific and technological cooperation project.

\section{References}

[1] S.S.P. Parkin, R.F.C. Farrow, R.F. Marks, A. Cebollada, G.R. Harp, R.J. Savoy, Phys. Rev. Lett. 72, 3718 (1994).

[2] S.S.P. Parkin, T. Rabedeau, Appl. Phys. Lett. 68, 1162 (1996).

[3] T. Luciński, F. Stobiecki, D. Elefant, D. Eckert, G. Reiss, B. Szymański, J. Dubowik, M. Schmidt, H. Rohrmann, K. Röl, J. Magn. Magn. Mater. 174, 192 (1997); T. Luciński, F. Stobiecki, B. Szymański, J. Dubowik, M. Schmidt, M. Urbaniak, J. Phys. IV 8, 453 (1998).

[4] J. Baszyński, F. Stobiecki, B. Szymański, K. Chrzumnicka, Phys. Status Solidi A 141, K23 (1994).

[5] D.B. Fulghum, R.E. Camley, Phys. Rev. B 52, 13435 (1995).

[6] F. Stobiecki, T. Luciński, C. Loch, J. Dubowik, B. Szymański, M. Urbaniak, K. Röll, J. Magn. Soc. Japan 23, 176 (1999). 